\title{
SANTIAGO LUZURIAGA Y SUS NOBELAS-KOMEDIAS EN LOS FONDOS DOCUMENTALES Y BIBLIOGRÁFICOS DE PATRIMONIO NACIONAL ${ }^{\alpha}$
}

\author{
Santiago Luzuriaga and his nobelas-komedias works \\ in the archival holdings and bibliographic collections \\ of Patrimonio Nacional
}

\section{Javier Fernández Fernández ${ }^{\circledR}$}

Fecha de recepción: 21/04/2020 • Fecha de aceptación: 07/10/2020

Resumen. En este texto se expone la biografía del maestro navarro Santiago Luzuriaga y Odria (1828-1904), que contó con una extensa carrera profesional y fue padre del pedagogo Lorenzo Luzuriaga. Santiago Luzuriaga fue el autor de unas obras singulares, escritas como agradecimiento por el hecho de que el Estado se hiciera cargo del pago a los maestros en 1901, que llamó nobelas-komedias. De estas obras se conservan algunos ejemplares en el Archivo General de Palacio y la Real Biblioteca. Estas novelas-comedias tienen la particularidad de que en ellas se empleó la ortografía fonética. Defendía así, el autor, la necesidad de transformar la escritura para progresar en la economía del lenguaje. Finalmente, se reproduce un ejemplar dedicado a Alfonso XIII de la obra El ermano del alkalde, conservado en la Real Biblioteca.

Palabras clave: Santiago Luzuriaga y Odria; biografías; ortografía fonética; Patrimonio Nacional; Lorenzo Luzuriaga Medina.

Abstract. This paper presents the biography of Santiago Luzuriaga y Odria (18281904), a teacher from Navarre (Spain), who enjoyed a long professional

\footnotetext{
a Quiero manifestar mi agradecimiento a Daniel Gozalbo Gimeno del Archivo General de la Administración por la ayuda en la localización de los expedientes de ese centro y a Nuria Lázaro Milla, sobrina nieta de un alumno de Recaredo Medina, por las revisiones del texto.

${ }^{\text {B }}$ Archivo General de Palacio, Patrimonio Nacional. Palacio Real, C/ Bailén, s/n, 28071 Madrid, España. javier.fernandez@patrimonionacional.es (D) https://orcid.org/0000-0003-3145-4569
}

Cómo citar este artículo: Fernández Fernández, Javier. «Santiago Luzuriaga y sus nobelas-komedias en los fondos documentales y bibliográficos de patrimonio nacional». Historia y Memoria de la Educación 13 (2021): 755-780 
career and was the father of the Spanish pedagogue Lorenzo Luzuriaga. Santiago Luzuriaga was the author of singular works, which he called nobelas-komedias, written after the Spanish Government agreed to start paying teachers' salaries beginning in 1901. The Archivo General de Palacio (General Archive of the Royal Palace of Madrid) and the Real Biblioteca (Spanish Royal Library) preserve some of these nobelas-komedias, which feature the peculiarity of being written with a phonetic spelling. The author argued for the necessary transformation of writing for the purpose of achieving a greater economy of language. Finally, the paper includes a copy of $\mathrm{El}$ ermano del alkalde dedicated to King Alfonso XIII of Spain, which is currently in the Real Biblioteca.

Keywords: Santiago Luzuriaga y Odria; biographies; phonetic spelling; Patrimonio Nacional; Lorenzo Luzuriaga Medina.

El Archivo General de Palacio y la Real Biblioteca cuentan con ricos fondos, y no lo suficientemente aprovechados, para realizar investigaciones en el ámbito de la historia de la educación. Ambos centros, situados en el Palacio Real de Madrid y gestionados por Patrimonio Nacional, atesoran documentación sobre distintas instituciones escolares desde el siglo XVI hasta el siglo XX. Unas dependieron directamente de la Real Casa; es el caso del Colegio del Monasterio de San Lorenzo de El Escorial, el Colegio menor de San Felipe y Santiago (o del Rey) en Alcalá de Henares, el Colegio de Santa Isabel, el Colegio de Nuestra Señora de Loreto, el Colegio de las salesas reales, la Casa de caballeros pajes, el Colegio de niños cantores, las Escuelas de niños y niñas de la Real Casa, el Asilo de niños de lavanderas o el Asilo de párvulos de María Cristina, en Madrid. En otros casos, los reyes subvencionaban la educación de colegiales, como en las Escuelas pías de San Fernando y San Antonio Abad o el Colegio de Nuestra Señora de la Presentación, conocido popularmente como de niñas de Leganés, en Madrid.

Asimismo, podemos localizar entre sus fondos documentos relacionados con la educación de miembros de la familia real. Abundan los correspondientes al período comprendido desde el nacimiento de la infanta Isabel de Borbón y Borbón en 1851 hasta el establecimiento de la Segunda República en 1931. Se conservan testimonios de la formación de la mencionada infanta, de su hermano el rey Alfonso XII y de sus dos esposas, María de las Mercedes de Orleans y Borbón y María Cristina de Habsburgo-Lorena, al igual que de su heredero Alfonso XIII y de los hijos de este: el príncipe Alfonso y los infantes Jaime, Beatriz, María 
Cristina, Juan y Gonzalo de Borbón y Battenberg. Ejemplo de esta documentación son los distintos métodos de aprendizaje de lectura conservados en la Real Biblioteca.

También encontramos entre los fondos bibliográficos y documentales de Patrimonio Nacional proyectos impulsados por los monarcas o sus familias, como los de la creación de escuelas en los reales sitios en tiempos de Carlos III o los planos del arquitecto José López Sallaberry del que debiera haber sido el primer grupo escolar de Madrid, costeado por la familia real e inaugurado en 1902 para conmemorar la mayoría de edad de Alfonso XIII. ${ }^{1}$ Y, por último, podemos localizar peticiones y adhesiones a los monarcas y su Casa solicitando o agradeciendo el apoyo de iniciativas de todo tipo, muchas de ellas relacionadas con el ámbito educativo. A este respecto, hemos podido ver recientemente en la exposición Madrid, ciudad educadora (1898-1938). Memoria de la escuela pública el álbum ofrecido por los alumnos de las Escuelas Aguirre a Alfonso XIII con motivo de los 25 años de reinado. ${ }^{2}$ Igualmente, entrarían en esta categoría el plan de edición del libro del profesor de primera enseñanza superior Eugenio Fernández del Corral, Única aritmética métrico-decimal, teórico-práctica para la Primera enseñanza, de 1881;3 el puzle-alfabeto que José Roca Ruscalleda, maestro de las escuelas públicas de Valencia y Barcelona, elaboró para que Alfonso XIII aprendiera a leer; ${ }^{4}$ o la petición de una joven Carmen Conde a la reina Victoria Eugenia en 1927 para que le concediese una pensión de estudio y poder dejar su empleo en la Sociedad Española de Construcción Naval. ${ }^{5}$ Como

\footnotetext{
1 José López Sallaberry, «Escuela graduada», 1902. Planos, Mapas y Dibujos, 1661-1663, Archivo General de Palacio (en adelante, AGP).

${ }^{2}$ Escuelas Aguirre, «Álbum ofrecido por los alumnos de las Escuelas Aguirre a S. M. el rey Alfonso XIII». Fotografía Histórica, 10124347, AGP.

${ }_{3}^{3}$ Eugenio Fernández del Corral, «Única aritmética métrico-decimal, teórico-práctica para la Primera enseñanza», 1881. Administración general, caja 12857 expediente 7, AGP.

${ }_{4}^{4}$ José Roca Ruscalleda, Leer jugando, dedicado a S.M. Alfonso XIII, mayo de 1888 (Barcelona: Imp. Juan Tarral, 1888). El folleto está dentro de una caja en forma de libro que incluye el rompecabezas y las fichas para aprender a leer. Real Biblioteca PAS/3808. Para una visión general de las colecciones de los infantes que conserva la Real Biblioteca recomiendo el artículo de Arantxa Domingo Malvadí, «Juegos con libros y libros de juegos en las bibliotecas de los infantes» en La fisonomía del libro medieval y moderno: entre la funcionalidad, la estética y la información, dir. Manuel José Pedraza Gracia (Zaragoza: Prensas de la Universidad de Zaragoza, 2019), 517-529.

${ }^{5}$ Carmen Conde Abellán, «Carta a la reina Victoria Eugenia», 1927. Administración general, caja 15278 expediente 1 , AGP.
} 
ejemplo muy significativo de documentos de apoyo a los monarcas queremos presentar unas breves «nobelas-komedias» que escribió y remitió a varios miembros de la familia real el maestro Santiago Luzuriaga y Odria, padre del pedagogo Lorenzo Luzuriaga.

\section{SANTIAGO LUZURIAGA Y ODRIA (1828-1904)}

Santiago Luzuriaga tuvo una larga carrera como maestro. Ejerció durante cerca de 50 años en multitud de escuelas, sobre todo de Navarra y País Vasco. Nació en el municipio navarro de Murieta, en la merindad de Estella, el 3 de mayo de 1828. Según su hoja de servicios, ${ }^{6}$ comenzó su andadura profesional en la Escuela elemental del municipio de Barbarin el 1 de agosto de 1853, después de haber obtenido el título de primera enseñanza elemental el 8 de junio de ese mismo año. A lo largo de once años estuvo ejerciendo en diferentes escuelas elementales navarras: Escuela pública de Barbarin (1853-1854), Escuela elemental incompleta de ambos sexos de Arzoz (1854-1857), Escuela pública de Azcona (1857-1860) y Escuela elemental de niños de Zubieta (1860-1864). En esta última localidad también ocupó el cargo de secretario del Ayuntamiento hasta noviembre de 1865.

Posteriormente, se trasladó a la provincia de Guipúzcoa, donde se incorporó a la Escuela de párvulos de Tolosa como director interino (1865-1866) y, por concurso, a la Escuela elemental completa de niños de Alzo (1866-1867). En marzo de 1867, retorna por concurso a Navarra, a la Escuela de párvulos de Estella, donde desempeñó su magisterio hasta 1880 . Tras el concurso celebrado el 15 de diciembre de 1869, recibió una mención honorífica de la Junta de Instrucción pública de Navarra por «su esmerado celo y satisfactorios resultados obtenidos en la enseñanza». Es en Estella cuando enviuda de Valentina Aguirre, madre de sus hijos Benito y Fermín. En 1880 se trasladó a la Escuela de párvulos del municipio vizcaíno de Bermeo como maestro en propiedad. Allí estuvo tres cursos académicos y se le expidió un certificado de la Junta local de primera enseñanza de reconocimiento de su labor «a completa satisfacción de autoridades y vecindario».

\footnotetext{
6 «Expediente personal de Santiago Luzuriaga y Odria». Educación, IDD (05)001.019, caja 31/18945 expediente 4, Archivo General de la Administración (en adelante, AGA). A la hora de tratar de su carrera profesional y su jubilación, si no citamos otra referencia nos referiremos a este expediente.
} 
En septiembre de 1883 cambió de aires y se trasladó a La Mancha. Ocupó por ascenso una plaza de maestro en propiedad en la Escuela pública elemental de párvulos de Valdepeñas (Ciudad Real), donde trabajó durante más de ocho años, hasta abril de 1892. Se casó en segundas nupcias el 12 de abril de 1888 en Socuéllamos con Ángeles Medina Moreno, ${ }^{7}$ también viuda. Ángeles era hermana del profesor Lorenzo Medina, compañero de Santiago y persona de grato recuerdo para la ciudad manchega. ${ }^{8}$ De este matrimonio nació el 29 de octubre de 1889 Lorenzo Luzuriaga, cuando Santiago contaba con 61 años y Ángeles 37. Ambos tenían hijos maestros: Fermín Luzuriaga y Recaredo Medina. ${ }^{9}$ Lorenzo Luzuriaga en unas notas autobiográficas se refería de este modo a la profesión mayoritaria de su familia: «Así, pues, he tenido padre maestro, dos tíos maestros y dos hermanos maestros; no se puede pedir más pedagogía». ${ }^{10}$

En 1892, Santiago se mudó más al sur, a Jerez de la Frontera (Cádiz), como director de la Escuela pública elemental de niños del distrito de San Fernando. Allí permaneció hasta septiembre de 1893, en que se trasladó como maestro de primera enseñanza a la Escuela de niños del tercer distrito de Bilbao. ${ }^{11}$

Contamos con información de sus últimos cursos como maestro. En el curso 1897-1898 la matrícula de su escuela era de 134 alumnos, con una asistencia media de 94 niños, y consiguió un 16\% de concesión de premios. Al curso siguiente, la matrícula bajó en 12 alumnos, pero la

\footnotetext{
7 «Expediente de clasificación de pensión de viudedad del magisterio de la Dirección General de Clases Pasivas en favor de Ángeles Medina Moreno». Hacienda, IDD (01)007.017, caja 62/02962, exp. 361, AGA.

${ }^{8}$ Uno de los primeros grupos escolares establecidos en Valdepeñas fue bautizado con su nombre en 1927 y todavía hoy pervive como centro de educación infantil y primaria. Véase Francisco Asensio Rubio, «La enseñanza en Valdepeñas: 1900-1939» en 3er ciclo de conferencias: Valdepeñas y su historia, Matías Barchino Pérez et al. (Valdepeñas: Ayuntamiento de Valdepeñas, 2008), 108.

${ }_{9}$ Su nombre completo era Recaredo Evelio Eladio Antonio Medina Medina. Compartía con su hermano Lorenzo el nombre de Eladio, pues éste recibió en la pila bautismal los nombres de Narciso Eladio Lorenzo.

10 «Don Lorenzo Luzuriaga», Negro sobre blanco: Boletín literario bibliográfico, 17 (1961): 1-2.

${ }^{11}$ En el expediente personal se indica que la escuela es la del 3er distrito, en cambio en algunas de las instancias de Luzuriaga consta que las escuelas son las del distrito $5 .^{\circ}$. Santiago Luzuriaga contaba entonces con 65 años y, según el censo electoral de Vizcaya de 1897, vivió en la calle Aréchaga, $7,2 .{ }^{\circ}$.
} 
media de asistentes se incrementó en 2 y consiguió un $18 \%$ de concesión de premios. En este último curso su escuela obtuvo la categoría «Tercera» y fue calificada como "Buena», como otras ocho escuelas bilbaínas. ${ }^{12}$ En septiembre de 1898, cuando tenía 70 años, elevó una instancia solicitando una licencia para ir a Madrid a «adquirir la preparación para cursar el tercer año para Maestro Superior».13

Sus últimos años de ejercicio profesional en la capital vizcaína fueron tensos con la Junta local de primera enseñanza de Bilbao y el resto de la administración educativa. Se iniciaba así un largo y penoso litigio que terminó con su jubilación. Santiago Luzuriaga achacaba sus problemas con la Junta local a la inquina personal que le profesaba su secretario, Manuel Agustino y Barco. Acusaba a Agustino de que siendo todavía maestro le invitó a una fiesta y con un canuto le tiró polvos negros a los ojos, «quedando casi ciego» de ambos. Finalmente, pudo recuperar la visión del ojo izquierdo, terminando "tuerto del derecho». Mientras se recuperaba, fue auxiliado en las tareas escolares por el hijo de su mujer, Recaredo Medina.

La Junta local, por su parte, le acusaba de varios cargos. En primer lugar, que la escuela se hallaba en perpetuo desorden por «la falta de organización y plan fijo». En segundo lugar, que dentro de las horas de clase había convertido la escuela en «teatro de ridículos espectáculos organizando carreras de competencia entre los niños, e imponiendo otros castigos impropios del lugar con relajamiento de la disciplina y desprestigio de la dignidad profesional». En tercer lugar, que la escuela tenía insuficientes o nulos resultados debido al «poco celo» que demostraba en bien de la enseñanza, al limitado ascendente que tenía sobre los niños y a la «escasa instrucción, aptitud y capacidad» de Santiago Luzuriaga. La queja fue elevada a la Junta provincial de instrucción pública de Vizcaya que incoó un expediente gubernativo.

\footnotetext{
12 Ayuntamiento de Bilbao. «Acuerdo por el que se declara enterado el Ayuntamiento del oficio de la Alcaldía poniendo en conocimiento del Pleno el resultado de los exámenes generales de fin del curso 1898-1899, del resultado y calificaciones otorgadas por la Junta local de primera enseñanza a los maestros responsables de las escuelas públicas de la villa y del estado de la matrícula y asistencia de niños a clase durante dicho curso elevado por la citada junta», 1899, Archivo Municipal de Bilbao. ES.48020.AMB-BUA 489974, Archivo Municipal de Bilbao.
}

13 «Expediente personal de Santiago Luzuriaga y Odria». AGA. Todo el proceso de jubilación se documenta en este expediente. 
A estos cargos respondió Luzuriaga en la última semana de 1898 que la organización de la escuela de mi cargo está relacionada con la clase y números de niños que a la misma concurren siendo la mayor parte de ellos procedentes de familias pobres y de poca educación que viven en las peores calles denominadas de Cantarranas y Miravilla [...] a lo que se debe agregar el penoso trabajo del profesor que tiene muy poco descanso porque no lo puede ayudar el Auxiliar D. Francisco Tejada por la crónica enfermedad que padece.

Consideraba el maestro que la escuela «está bien organizada como se ha demostrado en los exámenes generales que han sido aprobados todos los niños y se han distribuido los correspondientes premios». La segunda acusación, haber «convertido más de una vez mi escuela en teatro y que en la misma se han practicado carreras de copetencia [sic] entre niños mientras las horas de clase», la calificaba como calumnia. Y respecto a la acusación de falta de celo, respondió que «en Mayo y Junio último después de las horas de clase, di lección particular gratis en mi casa a mis discípulos lo mismo que lo verifico ahora por las noches costeando la luz, sobrellevando con gusto el trabajo».

El 22 de marzo de 1899, la Junta provincial elevó al rectorado de la Universidad de Valladolid el expediente gubernativo proponiendo la jubilación forzosa de Santiago Luzuriaga. El Consejo universitario comunicó a la Junta provincial que había resuelto se pidiese la jubilación o sustitución, «debiendo instruirse al efecto el expediente de jubilación por pasar el referido maestro de la edad de 60 años».

El 3 de enero de 1899 fue el propio Luzuriaga quien solicitó su retiro, pero al mes siguiente desistió por motivos económicos. Ante los incumplimientos por parte de Santiago Luzuriaga de la presentación de los documentos que apoyasen su petición de jubilación, el 26 de octubre de 1899 la Junta provincial se dirigió al Ministerio de Fomento suplicando decretase su retiro. Como el expediente seguía su trámite, la Junta provincial se volvió a dirigir al rector de la Universidad de Valladolid para que «resolviese la suspensión de empleo y medio sueldo» de Luzuriaga, «hasta tanto que le fuere concedida la jubilación». Finalmente, resolvió el rector que la Junta provincial nombrase un maestro sustituto. 
En 1900, la familia Luzuriaga Medina se traslada a la localidad madrileña de Aravaca, donde Recaredo Medina ${ }^{14}$ comienza a ejercer como maestro de niños y se convirtió en la principal fuente de ingresos de la familia. ${ }^{15}$ El 24 de noviembre de ese año, Santiago Luzuriaga solicita regresar a su escuela ya que

con las mil pesetas anuales que se le ofrecieron al decretar una arbitraria sostitucion [sic], no puede vivir con familia por lo que le tiene como de limosna en su casa un hijo político Maestro de Aravaca cuando todavía está útil para ejercer en su escuela, que legalmente adquirió en propiedad en Bilbao.

El 4 de diciembre de 1902, Luzuriaga exponía al subsecretario de Instrucción pública que había sido sustituido sin que le hubieran realizado un reconocimiento médico y sin consentimiento de la autoridad que le nombró, y solicitaba que se anulase su sustitución para poder remitir la certificación de los seis años que había ejercido en Bilbao, necesaria para su jubilación y clasificación.

Después de su relevo, Santiago Luzuriaga elevó varias instancias protestando porque la Junta provincial carecía de atribuciones para nombrar suplente o pedir su anulación. Dicha junta propuso el 1 de agosto de 1903 que se le declarara jubilado. El 20 de agosto de 1903, Luzuriaga elevó una nueva solicitud al ministro de Instrucción pública reiterando que su reemplazo no era conforme a derecho porque el sustituto «no tiene ninguna oposición aprobada, ni ha desempeñado en propiedad ninguna escuela pública». Además, acusaba a la Junta provincial de

\footnotetext{
${ }^{14}$ En 1907, Recaredo Medina Medina se trasladó al municipio de El Pardo donde ejerció hasta la Guerra Civil. Tras ésta, como consecuencia del proceso de depuración, fue inhabilitado para cargos de mando y de confianza y sufrió pérdida de los haberes no disfrutados. Falleció en El Pardo en 1942. Luis Bello visitó su escuela en 1926. En su crónica titulada «Corzos y niños en El Pardo», Bello hizo un elogio de la escuela y del propio Recaredo: «el maestro, señor don Recaredo, el cual está lejos de sospechar el profundo sentimiento de envidia retrospectiva que nos invade al cruzar la escuela y poner los pies en el patio-jardín. Si yo fuera chico de la escuela -iinquietante idea la de volver a empezar otra vez!-, ¡cómo me gustaría venir a este rincón de El Pardo! He aquí un maestro apacible y feliz. No tiene correas, ni caña, ni palmeta. Seguro estoy que no sabe tener mal genio [...]» V. Bello, Luis. "Corzos y niños en El Pardo», en Viaje por las escuelas de Madrid, ed. Agustín Escolano (Madrid: Consejería de Educación y Cultura, 1997) 99.

${ }^{15}$ Cuenta Herminio Barreiro que Lorenzo Luzuriaga terminó sus estudios primarios en Aravaca y que fue Recaredo quien le pagó los estudios de Magisterio en la Escuela normal central de Madrid. Herminio Barreiro, «Lorenzo Luzuriaga: una biografía truncada (1889-1959)», Sarmiento, 15 (2011): 183.
} 
tener inquina personal ya que en Vizcaya «ejercen muchísimos Maestros mas viejos, a los que no se les molesta, y por una injusta venganza personal, hace tiempo que es perseguido un desgraciado Maestro». Por último, señalaba que debía ser sustituido por la misma autoridad que lo había nombrado. Por todo lo anterior, suplicaba que se anulase su relevo, «previo Informe ó consulta del Consejo de Instrucción pública, para que el recurrente sea igual en sus derechos, á todos los Señores Maestros, que piden su jubilación». Finalmente, el 29 de agosto de 1903 el subsecretario 1. ${ }^{\circ}$ de Enseñanza y Escuelas normales firmó la real orden de jubilación comunicándosela al rector de la Universidad de Valladolid.

El 21 de mayo de 1904, Santiago Luzuriaga volvió a presentar una instancia al ministro de Instrucción pública porque no le habían contestado a las anteriores. El dictamen del Consejo de Instrucción pública se redactó el 4 de julio de 1904. La sección 1ª decidió desestimar la pretensión de reingreso. El 16 de julio la subsecretaría se dirigió al gobernador civil de Madrid para que remitiese a Santiago Luzuriaga la decisión. Unos meses más tarde, el 22 de octubre, Santiago Luzuriaga Odria falleció en su domicilio de la calle del Caño, n. ${ }^{\circ} 2$ de Aravaca. ${ }^{16}$

\section{LAS NOBELAS-KOMEDIAS DE SANTIAGO LUZURIAGA}

Es en estos últimos años de Aravaca cuando el padre de Lorenzo Luzuriaga autoedita una serie de pequeñas obras, ${ }^{17}$ llamadas por él nobelas-komedias. En Madrid conservamos tres de estas obras: El ermano del alkalde, Maniobras y La lebita i el baston del alkalde. Este conjunto de textos de temas costumbristas, jocosos y con trasfondo moral es el regalo que Santiago Luzuriaga ofreció a la Real Casa y a «los ocho Ministerios, i de todos los ke se an interesado, para ke kobremos kon puntualidad eskasos aberes, los Maestros de eskuela». El autor se refiere con esto al Real decreto, de 26 de octubre de 1901, autorizando al ministro de Instrucción pública y Bellas Artes para que en los presupuestos generales de su departamento incluyese las partidas necesarias para el pago de

\footnotetext{
16 «Expediente de clasificación de pensión de viudedad del magisterio de la Dirección General de Clases Pasivas en favor de Ángeles Medina Moreno», AGA. El domicilio correspondía con la escuela de niños según Facundo Cañada, Plano de Madrid y pueblos colindantes al empezar el siglo XX (Madrid: Mateu, 1900).

${ }^{17}$ Según el propio Santiago Luzuriaga la serie se componía de cuatro títulos, pero no indica cuáles.
} 
las atenciones de personal y material de las escuelas públicas de primera enseñanza. Este decreto supuso no sólo asegurar el cobro de los salarios de los maestros, sino que también el maestro pasó a convertirse en funcionario del Estado.

Santiago Luzuriaga escribió a la reina María Cristina pidiendo su amparo para publicar sus nobelas-komedias en octubre de 1901

para ke se pueda publicar en la prensa la segunda edición de las dos adjuntas Novelas komedias, i también para ke se publike la adjunta Memoria dedicada a sus Magestades, con sucesos supuestos de los Ministerios de la Presidencia y Gobernación. Las Novelas litografiadas, kon bastante informalidad, las han leído en los Ministerios respectivos, y en tertulias en donde les ha sido agradable la lectura, porke es moral y en medio de algunos chistes, se hace respetar á la Autoridad, por lo ke se separa el eskrito, de otras komedias perturbadoras, é inmorales. ${ }^{18}$

En el Archivo General de Palacio se conserva un ejemplar de El ermano del alkalde, que tiene la anotación manuscrita a grafito "Aravaca = Maestro de niños de». ${ }^{19}$ De este título, la Real Biblioteca guarda dos ejemplares dedicados por el autor a Alfonso XIII. ${ }^{20}$ De Maniobras, cuyo anterior título era, según su autor, Artilleros y tartamudos, la Real Biblioteca conserva dos ejemplares, ofrecido uno a la entonces princesa de Asturias María de las Mercedes de Borbón y Austria, ${ }^{21}$ y el segundo a su marido, Carlos de Borbón-Dos Sicilias. ${ }^{22}$

\footnotetext{
18 Santiago Luzuriaga, «Carta a la reina María Cristina», 7 de enero de 1902. Administración general, caja 13142, AGP. La carta conservada está fechada en 1902, en ella Luzuriaga dice que vuelve a mandar la solicitud por haberse perdido la que envió en octubre. En la misiva Santiago Luzuriaga solicitaba también una reunión con Alfonso Aguilar, secretario particular de la reina, para tratar de este asunto. En la carta desde la Secretaría particular de la reina María Cristina se anotó «Devueltas las comedias al interesado diciéndole que puede dirijirse directamente al Sr. Intendente [general de la Real Casa y Patrimonio] fecho 14/1/902».

19 Archivo General de Palacio, Biblioteca auxiliar, folleto 935. Los ejemplares conservados bien pudieron haber llegado en la carta que dirigió al Intendente general de la Real Casa y Patrimonio.

${ }^{20}$ Real Biblioteca, caj/foll4/29 (39) y caj/foll4/130 (2).

${ }^{21}$ Real Biblioteca, caj/foll4/46 (12).

${ }^{22}$ Real Biblioteca, caj/foll4/29 (38).
} 
La lebita $\mathrm{i}$ el baston del Alkalde, ${ }^{23}$ custodiada en la Biblioteca Nacional de España, fue editada por Valero Díaz en Madrid en 1903. Pero las otras dos se imprimieron sin fechar y mediante litografía en el propio domicilio de la familia Luzuriaga Medina en Aravaca. Estas novelas-comedias conservadas en los fondos de Patrimonio Nacional no eran las primeras que escribió Santiago Luzuriaga.

Lo primero que llama la atención de estas nobelas-komedias es que están impresas en ortografía fonética. Se suprimían siete letras: la c y la $q$ eran sustituidas por la $k$ para los sonidos fuertes y por la $z$ en los suaves; la $v$ era cambiada por la $b$; la $y$ por la $i$; la $h$, eliminada por su falta de sonido; la doble $r$ se escribiría con diéresis y la $x$ era reemplazada por $s k$. Santiago Luzuriaga contabilizaba catorce letras porque sumaba también las mayúsculas. Él mismo escribe en el prólogo de El ermano del alkalde que su objetivo «es el de kompetir kon otros Autores, ke han publikado en la prensa sus obras suprimiendo tres ó cuatro letras» y que

en akostumbrándose, se puede escribir i leer kon toda claridad, el kastellano, el katalán, el baskuenze, etz., sin faltar á la pronunziazión, ni perjudicar á nadie, en una época ke en todo se adelanta, a eszepzión de encontrar una razonada Ortografía, para que los ofizinistas, periodistas i eskritores de todas klases, no tengan ke konsultar Dikzionarios ke se kontradizen.

Estas novelas-comedias nos invitan a pensar que eran unas prácticas habituales que realizaba con sus alumnos. Deben relacionarse, asimismo, con la segunda acusación vertida por la Junta local de primera enseñanza de Bilbao. Más de veinte años antes de que litografiase estas obras, se editó en la logroñesa imprenta y librería de El Riojano una obra titulada Defensa e invenciones (1879), ${ }^{24}$ que contiene una "Novela equivalente a un drama. Travesuras y petardos. Drama en tres actos». Aunque esta obra utiliza la ortografía alfabética, reproduce una carta de Santiago Luzuriaga al director del periódico El Harense de 15 de septiembre

\footnotetext{
${ }^{23}$ Que lleva por subtítulo «Nobela komedia en un akto, i dos kuadros; dedikada á la Intendenzia i Maiordomía de la Real Kasa, en obsekio de los Ministerios de Grazia i Justizia i de Azienda».

${ }^{24}$ Se conserva un ejemplar en la Biblioteca del Instituto de Estudios Riojanos, signatura AM/2126. Como muestra del compromiso educativo de Santiago Luzuriaga, esta obra incluye una advertencia en la que el autor anuncia que «después de pagar los gastos de impresión, dará lo sobrante á algún estudiante que no tenga recursos ó a la casa de Beneficencia».
} 
de 1878, en la que el maestro defiende la eliminación de varias letras para que «encuentren algun descanso los muchos que luchan y sudan para enseñar y aprender en la época en la que otras ciencias han adelantado, $[\ldots]$ y para que tambien (por ilusion) puedan descansar los desgraciados sordo-mudos».

En la carta que Santiago Luzuriaga envió el 15 de enero de 1902 a Alfonso Aguilar, secretario particular de la reina María Cristina, el maestro exponía que era necesario reformar la ortografía en base a la pronunciación y así disminuir el uso de las letras: «No se puede negar que en España hemos progresado en todo, á escepción del huso [sic] de las letras, y por economía de tiempo y del indispensable material, repito que con el tiempo si la Academia quiere, podría haber muchos adelantos entre los que enseñan y aprenden, sin perjudicar a nadie». ${ }^{25}$

Santiago Luzuriaga no fue el único maestro que intentó simplificar el uso del alfabeto en España. A lo largo del siglo XIX fueron varios los textos que defendían la implantación en España de la ortografía fonológica con el fin de facilitar el aprendizaje y la extensión de la lengua escrita impulsados desde el mundo educativo. En 1839, el profesor de gramática de la Escuela normal de Madrid, Mariano de Rementería, publicó Conferencias gramaticales sobre la lengua castellana. En ella, el profesor defendía la simplificación de la ortografía ya que «en la escritura alfabética las letras no son mas que unos signos con que se representan los sonidos que componen las voces, y porque estos signos deben ser sencillos en todo lo posible». Siguiendo este principio conseguiríamos una «ortografía natural, simple y perceptible a toda clase de personas».26

En 1843, el impulso partió de la Academia literaria y científica de profesores de instrucción primaria de Madrid. En esa fecha se empezó a aplicar en escuelas asociadas a esta Academia una reforma ortográfica despreciando la etimología:

Atribuían a la $c$ en todos los casos su sonido gutural, traspasaban a la $z$ el sonido suave de la $c e, c i$, resolvían la $x$, eliminaban

\footnotetext{
${ }^{25}$ Santiago Luzuriaga, "Carta a Alfonso Aguilar, secretario particular de la reina María Cristina», 1902. Administración general, caja 13142, AGP.

${ }^{26}$ Mariano de Rementería, Conferencias gramaticales sobre la lengua castellana, ó, Elementos esplanados de ella (Madrid: Imp. de Ferrer y Compañía, 1839), 293-294.
} 
del alfabeto las letras $h, v, q, x, k$, como no significativas o duplicadas, inventaron un nuevo carácter $\check{r}$, para la duplicación de esta letra. $^{27}$

Este intento de la Academia de profesores provocó una reacción contundente por parte del gobierno. Se expidió una Real orden, con fecha 25 de abril de 1844, que estableció como texto oficial para las escuelas la obra de la Real academia española Prontuario de ortografía de la lengua castellana. Los maestros que no siguieran la ortografía académica podían ser suspendidos de su magisterio.

En las décadas siguientes se sucedieron los intentos desde el mundo de la enseñanza contra la ortografía de la Real academia española que Santiago Luzuriaga debió de conocer. Mariano Cubí y Soler, profesor de éxito en Cuba, México y Estados Unidos, publicó en 1852 A la nación española sobre reformas ortográficas. En la década siguiente, el inspector de primera enseñanza de la provincia de Lérida, Rafael Monroy, editó en 1865 Estudios ortográfico-prosódicos sobre la reforma que admite la escritura y pronunciación castellana. Diez años después, fue el regente de la Escuela normal superior de Ciudad Real, Francisco Ruiz Morote, quien publicó Ortografía castellana teórico-práctica. En 1889, el catedrático del Instituto de Bilbao, Tomás Escriche y Mieg, imprimió Reforma de la ortografía castellana, donde calificaba como «uno de los mayores absurdos, corriente como la cosa más natural en todos los países, con el beneplácito de las personas doctas, es el hecho de no escribir las palabras exactamente como se pronuncian». ${ }^{28}$

La profesora Mercedes Quilis Merín considera que el fin último de todos estos autores y algunos más que publicaron obras en años posteriores ${ }^{29}$ era

lograr la perfección y sencillez en relación con la pronunciación aludidas en el sistema de escritura del español; pero está orientada,

\footnotetext{
${ }^{27}$ Citado en Mercedes Quilis Merín, «La presencia de los neógrafos en la lexicografía del siglo XIX», en Gramma-Temas 3: España y Portugal en la tradición gramatical, eds. Marina A. Maquieira Rodríguez y María Dolores Martínez Gavilán (León: Universidad, 2008), 271.

${ }^{28}$ Tomás Escriche y Mieg, Reforma de la ortografía castellana (Madrid: Manuel Ginés Hernández), 5.

29 Onofre Peligro y Valle, Nueva ortografía del idioma castellano (Badajoz: Estab. Tip. de La Minerva Extremeña, 1905) o Agustina González López, Idearium futurismo (Granada: Paulino Ventura Traveset, 1916).
} 
además, a facilitar el aprendizaje de la lengua escrita y la lectura de acuerdo con principios de universalización y democratización de los conocimientos asociados a principios de matiz liberal en lo político, por la creencia de que, por la vía de la simplificación de la ortografía, se facilitaría el correcto aprendizaje de la lengua. ${ }^{30}$

\section{El ermano del alkalde}

La novela-comedia, costumbrista de trasfondo moral y con final feliz, se compone de un único acto. Los protagonistas son el alcalde, del que desconocemos su nombre; su esposa, Justa; Román, el hermano del alcalde; Cecilia, la criada; el alguacil, Cirilo Ruiz; la esposa de este, María; y el médico. El acto tiene lugar en el domicilio del alcalde y Justa. El autor incluyó algunas referencias a algunos de los lugares donde ejerció su magisterio. Así, Cirilo Ruiz era oriundo de Jerez de la Frontera y el vino de Valdepeñas tiene un lugar destacado en la recuperación del desmayo que sufre María.

Santiago Luzuriaga también ideó un sistema para que el apuntador diera órdenes a los actores utilizando los dedos de las manos:

i en esta komedia á la esposa del Alkalde, á la kriada, i a la Alguazila, (ke lleban otros nombres), se les markará el trabajo lebantando respectibamente, los dedos pulgar índize i anular de la mano derecha, i kon los de la izquierda, también respectivamente, al Alkalde, á su ermano, al Alguazil, i al Médiko, kon el pulgar, índize, anular, i meñike, de dicha izkierda.

30 Quilis, «La presencia de los neógrafos en la lexicografía del siglo XIX», 269. 


\section{REFERENCIAS}

Asensio Rubio, Francisco. «La enseñanza en Valdepeñas: 1900-1939». En 3. er ciclo de conferencias: Valdepeñas y su historia, Matías Barchino Pérez et al., 89-232. Valdepeñas: Ayuntamiento de Valdepeñas, 2008.

Barreiro, Herminio. «Lorenzo Luzuriaga: una biografía truncada (1889-1959)». Sarmiento, 15 (2011): 181-190.

Bello, Luis. «Corzos y niños en El Pardo». En Viaje por las escuelas de Madrid, editado por Agustín Escolano, 98-101. Madrid: Consejería de Educación y Cultura, 1997.

Cañada, Facundo. Plano de Madrid y pueblos colindantes al empezar el siglo XX. Madrid: Mateu, 1900.

Domingo Malvadí, Arantxa. «Juegos con libros y libros de juegos en las bibliotecas de los infantes». En La fisonomía del libro medieval y moderno: entre la funcionalidad, la estética y la información, dir. Manuel José Pedraza Gracia, 517-529. Zaragoza: Prensas de la Universidad de Zaragoza, 2019.

«Don Lorenzo Luzuriaga», Negro sobre blanco: Boletín literario bibliográfico, 17 (1961): 1-2.

Escriche y Mieg, Tomás. Reforma de la ortografía castellana. Madrid: Manuel Ginés Hernández.

Quilis Merín, Mercedes. «La presencia de los neógrafos en la lexicografía del siglo XIX». En Gramma-Temas 3: España y Portugal en la tradición gramatical, eds. Marina A. Maquieira Rodríguez y María Dolores Martínez Gavilán, 267-293. León: Universidad, 2008.

Rementería, Mariano de. Conferencias gramaticales sobre la lengua castellana, ó, Elementos esplanados de ella. Madrid: Imp. de Ferrer y Compañía, 1839. 
El ermano del alkalde. Patrimonio Nacional. Real Biblioteca, caj/foll4/29 (39)
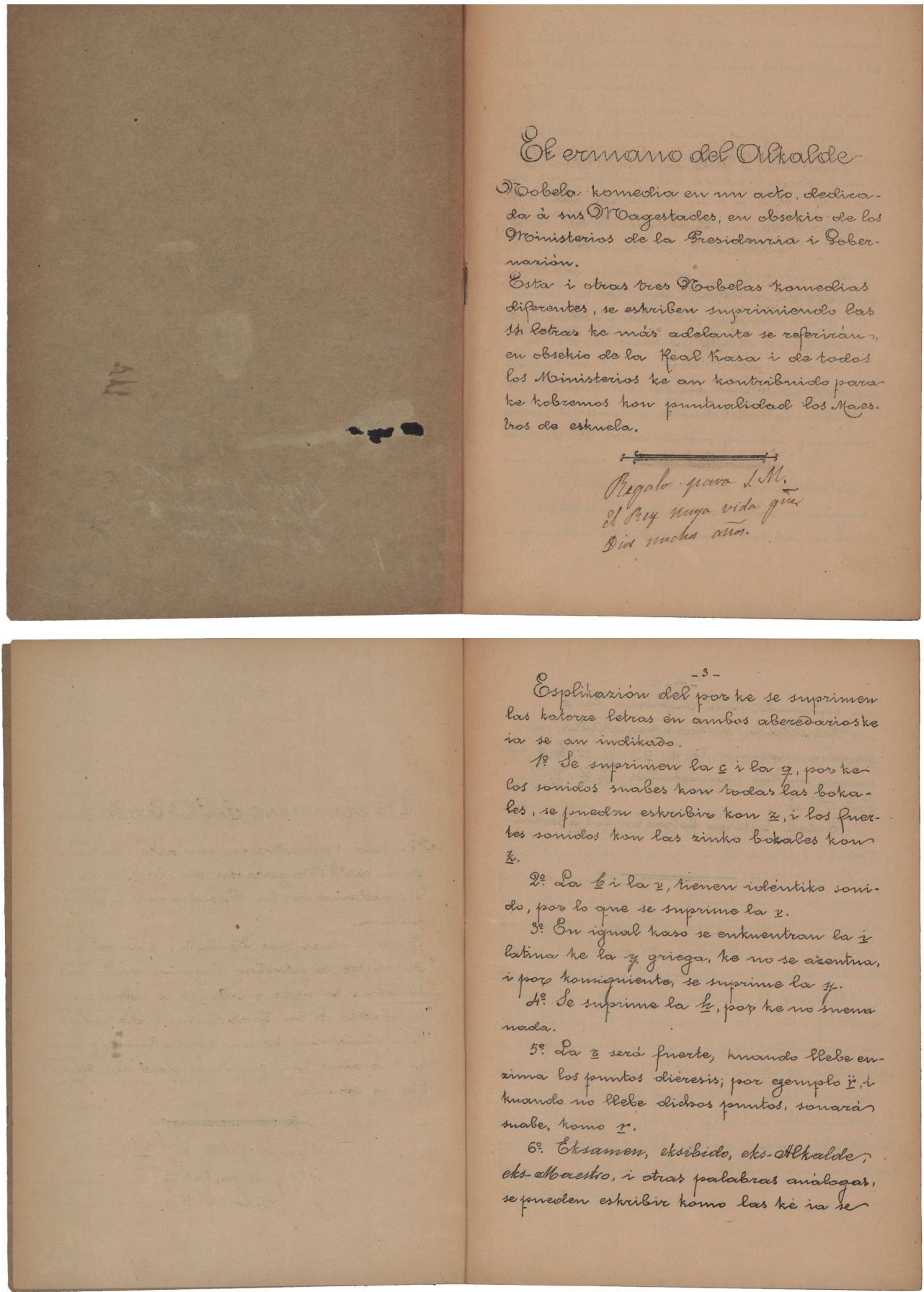
an crpressads, para smprimir la $x$.

Resnltado: en las mimistunlaxs, se

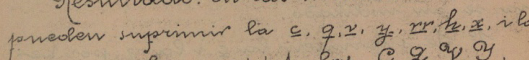
mismo en lous mainsknclas $\mathcal{C}, \mathcal{Q}, \underline{Q}, \underline{Y}$ $\mathbb{R}, \mathcal{H E}, \underline{X}$

Botal, tatarne latras en los abexodarios

Por este rarzomado mátools, em min poko tiemps, se padici aprentoer lo one és indispensable saber, para los usos Kannues de la bida; se ekanamira tien po, papel, tinta, etra, it toolas mas podrernas entender mas klaro ke asta avora.

Las ke kieran, pnevlen kö̈egir los eskritos the se on referivolo, para ke bean the en lo esensial, no oi onfercenaina $y$ ke se prede estribir y leer kan tada klaridad. empleando los signos de puntuarion, he aora se acostimubra.

Qtra imbenkión del ke sustri. Ge, tansiste en the los kámikas kaw el apum. tadar, se paneien entender par las serñas de los decolos, iv en estar hamediar a la espasa del Oulkaldo, à la kriada, is a la Olgma. sila, (ke lloban atros nambres), se les mar tarara el trabajo libantando respectibannewle, los declos pbnlgar indize i ammlar de la mano derectra, is kan los de la iratierdas también respektibannante, al Olkalder,

$$
\text { - Prólogo- }
$$

Ol puplikar las knatro 950. belos-Nomedias ke se on referiodo, no se intentar faltar al respeto de ningmos

El olgeto, es el obe kompetir kom ohos Ontores, tre am publitrado en la prensar sus obras smprimienolo bres : knua tro letras, en krada abexedario, ir en am. bos, of the suskribe. smprinne las katurace letras the se on aemastrowlo; $i$ sin embar. go, en akastumbriondase, se pmeote eskribir $i$ leer kan toola klovinolad, el kastellamo, el tratalion, el bastinense, etra, sin faltar à lar prommitianión, nis perpnolicar à ma. die, en mor époka ke en toobo se adelanta, a esxeprión de encontrar una rationa. da Ortagrafía, para que los ofirimistas, periodistars i eskritares de todols hlases, no tengan tve kanonltar Dikxianorios then se Kantradirien.

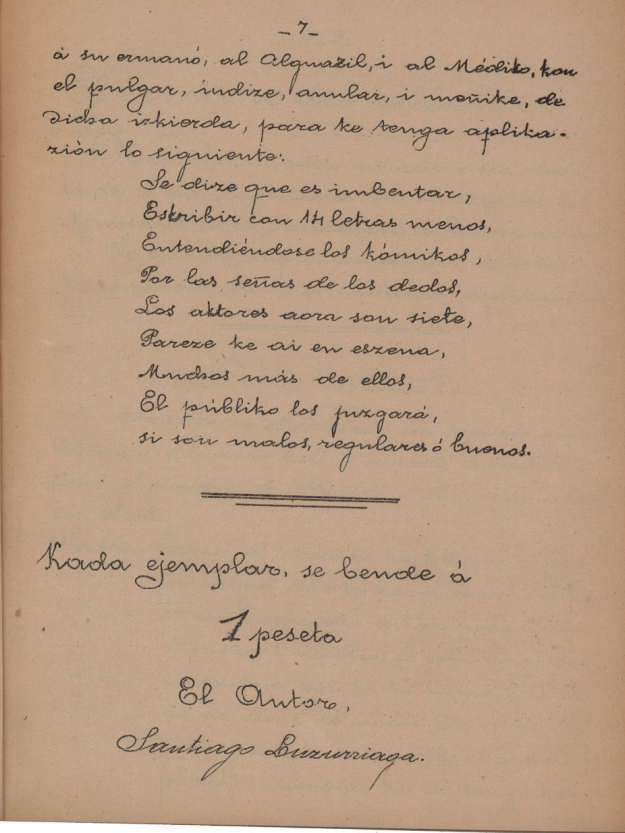


$-9$

Ol ermano del Olkabole.

O) Gobela-hamedia en mn atito, deoli. trada a sus Magestowles, on absekio de las Ninisterias do La Presidensia i 9abermaxión

Oe estaribe smprimiendo las 11 le letas the se refieren en la prevedente esplikaricin.

Onto imition

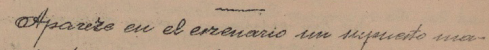

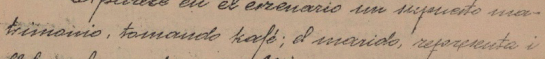

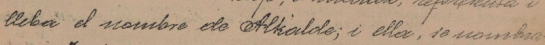
ta etrusta.

Orusta. Esposo, el Noidiko he bibe en el pito prinaspal de esta hasa, dize the el ka fé es un remredio ofikar para kitiour of snerino; i nossotros, lo tomamas par. meresidacid, par ke tiv twano Galkal. ole, de dia $i$ de nache tiemes twe bir. gilar para ke se knomorbe al ordem pinbliko, unientross the is, tengo ke

\section{-10.}

esperaro sin olamine, asta ke tow re. greses dal serbiriv.

Alkalda. In te punedes akostar y ke me espere Lerilia ha kriada.

tustas. En la kriada, no podemos temers kompianna; lo digo, par lo ke tengo obserbado.

Qfustas. Mira, esposo; the ermano Remianeb potografó, biene tarde muchas noches oi kasor; i si na me ohverma, sen olverme también la kriacla, par lo twe tow ermans rara despertames, despones de pagar aldabaros, tiener twe tirar tanitos a los balkanes, para despertarnos; asi es the estion ratos $w$. nos mantos tristales, itw tramo of. kalde, ir ano propietoric de esta ka. san, trenes ke deris taien à de pagarn los kristalos the estan notos.

Qthealde. He los pagno mi ermano Remín. paro ke biene tarde a karai pures no as justo the the mi ha kriada mi is paqnemos los bidrias rated.

\section{$-11$.}

Starta. Bi th ermano no tiena un sénti. mo, par lo twe are tiampo tre ande pegan. do sablaraf; io, sin ke tw lo sepas, le é deaso algmnos tanoutos, i del puppilage, nne debe ols monsmalidowes, $i$ entor esperando at he trabreis los 12.000 oluras the abeis eredado de bnestro tio Aaco, el folverón ke mnio en la amiériea.

(Eutro la tricacla).

Hriada Señor, mo he biste de kaballero, dine ke tieno kre ablar kan usteo.

Olkadla. Mira Kexulia, Hita estos serbizios del ka. fi, deja la batella del agna i di al he biste de kaballero he pase.

inicala. Estai bieni kitaré estos serbivios i kmm. plivé kon la aroen. (Sache kan-lat sertixial). Tusta. Siempro sera alguno the se bieve is nejar, ó algun embajasars, hon algu.

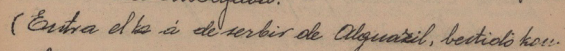
dereviria i how sandereso de kopa ev la mano). Alonacil. Anenas noches tengan sus Merrodes. Me. à dideo mi mpeniar, ke entreque 


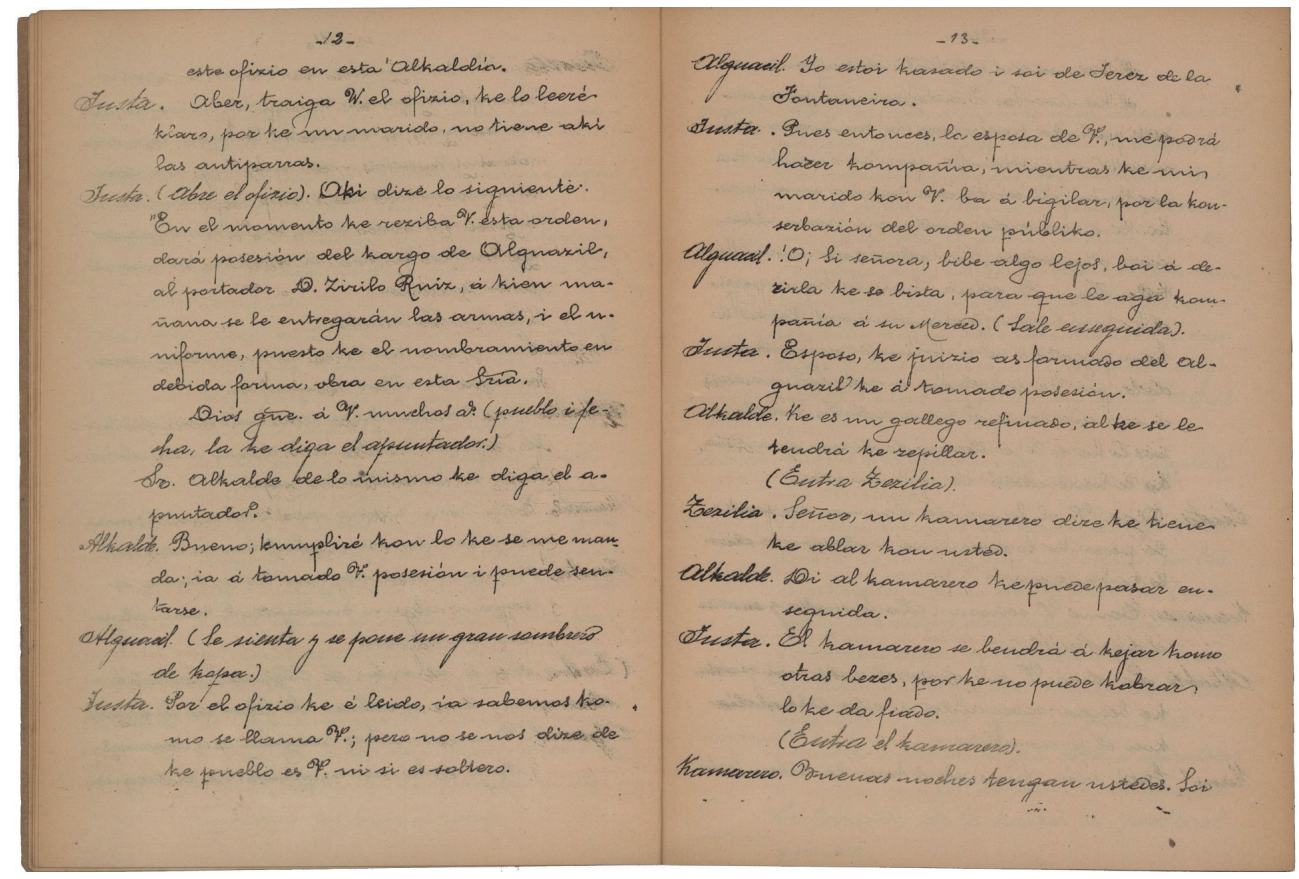

\footnotetext{
$-14-$

at tramarrero del kafe de enfente, en el ke un lo. a entregaso en porendar este pakete he parexe he trantiense dohn. mentos impartantes, por he a dichs tie. notenia olivero para pagar ma pese. ta ke a' echo de gasto, ni para abonar otra peseta, par aber rioto nica botella llena de agua, taon la ta e a perjudikado à bariol parracinand, los kele hau pegavo is se à formado nu eskáw. dalo, por ke tambitiu a roto las warires a'un ansiano, el he is roto la botellar, parloke sele à llamads à un quardia. the letiene atado al delinkunente.

thista Oaga op. el fava de darme ese pake. te para the lo evamine i desponies diré lo ke kantiene.

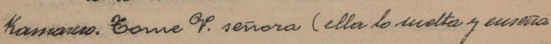
of hortevidas.

Clhalde. Le olira' OF, al gnardia de mi parte. the benga ensegunda is esta Olkaldia kan el preso atado.

Kam! Está trién; tammpliné tran la orden. (tale).
}

\section{$-15$.}

Quntá. Hira, esproso, nivica lo opre ai en esten packete; troplocs ke bewien los miegos, ba rias retratos, entre los que estion el the. io i el mio. Esto no se puses talerar,

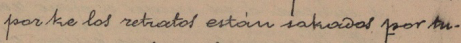
ermans, $i$ el ke benoe los retratos, tam bien será haparr de benoer las arigina les; $i$ th transo oltwalce, tienes the has. trigar à tre emrano, ó no terrarís dig. nidas, hamo marido, ni camo Oltalae.

altadse. Thes the kieres, mujer, ithe io pegno a mi emano?

Orusta. Ori otrol medios de kastigar; ia beras tromo is lo ago, por un ofirio, the bai a estaribir, itur ls as ole firmar (zepo-

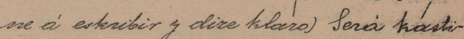
gads how rifor en la respectiba har. rel piblika, el ke sea tramourides kow esterofinis. Dios iñe a op. ms as. (puebto i fecha, tho ke diga da apastatain) El Alkoxtoe he nombrarás So. Olknide, de la karrel de, foueble, el que sea. 


\section{6.}

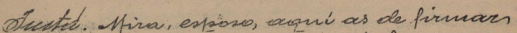
idespries, we entregariés firmado, sePlads i ser̈ars, el ofirio.

Ofthadale. Qrie lo the manda mi esposal lo axe.

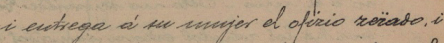
ella to quarder ex un kajain, o'en el bolivillo.)

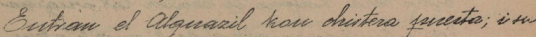

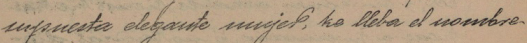
ae pibanial.

Navia. Imenos noches tengoun sus Merrodes.

Ollicalde. Eengan instedes mui buenas.

alguasil. Esta es mi señona, he biene à arar-

le kompañia à la dra Othaldesa.

ofthalde. He sca biem henciala. Faratroles, ithe

groysa es la mijer de ini Olgua.

Oruta. Para ti, todas las mineres son qua.

pass, pers es mas he ta ke is bencido?

la ke gobienna i manioa en tur kasa.

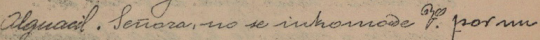

pirapm que su maridu le à echa. dn à uni nuios.

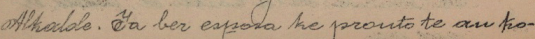

\section{$-13$}

noxido the the eres mu tormento en es ta kasa.

Havia. Aevares, io, no kisiera he por ini se tengan dizgustos, mi soyjechas en estha hasor.

Wthadide. 960 los tenarer ws, par the is, ive

pronto à knmistirs tear mi obliga.

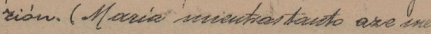
dia, ha saka del bahillo 's arracto ase mesic

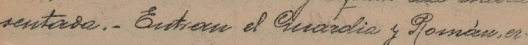

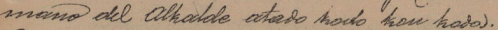

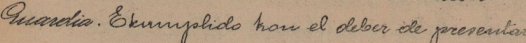
en esta alkablia os evte preso, al tra be kitoci: el kardel, pona atan à otro. (selolititu Arivion. Or. Onaralias hon exte ofirio, tendra O. We handwir al poreso a la reypeti. ba kanmel.

Inocidia. Obo pundo trandeszender, por ke then go he esiar end punto tre se me ida. signado por mi regoehtivo Iofe; i oin

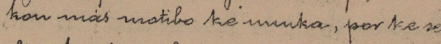
dine the a de aber nolga is mamifesta. siow, paro konsigninente, kredenoe inste. des haw Dios. (Sale)

Prista. Gers ambre Romon poro he no bienes tempramo a keasa, para he los tres the so. mas' de familia onirado, no pasemos bergiverna ke ir estamas pastando.

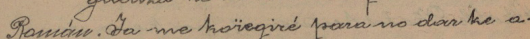
blar ni prasar la beroinenra he oir is parade, Inands me segnian los chitios oritands le lleban poreso is ataso al er. mans del Glkable, algin selits alarí Daso.

Oresta. Si tru no mbieras dado mativo, no te mbiesen gritado.

Pancian. Ato kres ke no es delito el ramper nna botella in kerer, ni el dar thomo prenda, unos detimmentes asta ke se purioa pagar mua peseta der gasto.

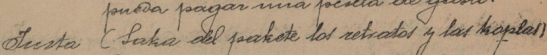
Law estas las olohmmentos ke as eimpienoso?

Peanáa. Li, san los mismos, io los tería bender i no é podindo.

xhadde. Sribonaro; as tenido balor pära emperiar i bender los retratos die la

$$
-19 .
$$

kasov de tiv ermoms?

Pramain. (oe radillas) Seroaname th ke tienes antarialave, kramo ilkabder i kamo er. mano miaira de la kasa.

altcalde. Tnes par eso, no te priedo perdanar lebantate ipoikrita.

Panam. (He lebantas $i$ are he hora).

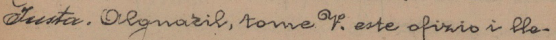
be ensegnida á este preso a la harrel. Panian. (Bbarando). The poko trompasion tirewen aki de min pobre fotiografo, pan ke à kerrolo bender mos retratos ke

los iro fiados i no los a kobrado, iré aila harrac haw al Olgnaxil, pero smphtio ef ta no se me handurka arado para ker no se oliga ke he Sheban pross al ermano olel oulkable.

orusta. Eypera un patso, knminado, the te boir à bracer la ropor is sinko pesetors para los primeros goestos. (Ba oc scliri).

esthable. A mi me tracicis al trage de nodser i la bara de Ockalde.

cresta. Bnens, serin serkidos los riss erma 
mos. (Socle)

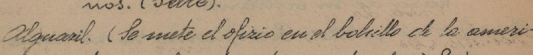

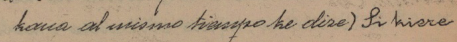
et preso he se la llebe a la karral sin o. tave, à de ser à kandizión des ke wa se. ha dhe eskapare.

Spancium, Doi formal palabra de que no me eseapocré.

Chicalde. If is the eshapas, of Olgmanil $y$ thw se. reis responsables.

Tresta. Forma Romam la kapa i nu dura para ef biage.

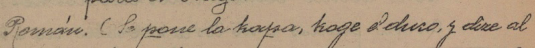
Aloriarid iva estamal andombes, asta Ple. Gar à ha kavroses (Salar hos dos).

Tusta. En espoto, ben akir, kie te boi a motern

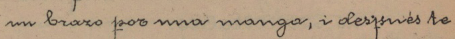
entregari ha bara de Alkalde. (Be caim. cha à bestir á su inarido, í lo da la basa).

Olkatbe. Oya já, i ke majo estoi rebestido der ountoriohoo; avera bai a recoirer la pablacion prova the se kanserbe el arden pain.
blike.

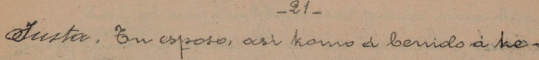
jarse ol kamarers, priese benir à kejarse hamo obras bexes mav nujer porke le à peyerso an maridosma palivas is mnna rido à derin que sw munger he à bendide los unebles i se as wlo as bibir kan atro. Lnego proven kejarse por ke en tal kalle ais unar riña of en otro sitio un inienDio; esta hat in io tolas itwe les piremosa las ke se biemen a kejour?

Athalde. Decir id todos los the te kioran kejar, no está el Ockelde en thater: Boir à emmplir kou mi obligarion, por ke se me aske tarae. (Pale).

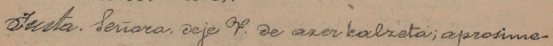
spl, ir sentordas, teriarenos un rato der charla.

Hoxia. He exprovinna kow su ariento.)

Thestar. held pareve a Op. lo ke fou los ombres; mi marialo, todlas has noches me aeja talo, aire que barainecorier la poliarión y el Diablo mabra' lo ke are mini espeno despmes the se

\section{$-22-$}

Menia. Señaras no sea Th. selata niv mal pena

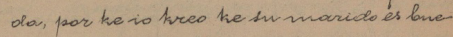
no i nu kaballero.

diactes ithan ke bneno, e? $i$ en uni presenvia le à echaso à of nuretricbro in no se lo ke prodria ares en mi ausenxia, trow ha hapar re ke es bmeno.

Hazia. Bo que une d' dicho nu marich, no es nasa i wi no of: a podido abserbar he uni maride no se a inkanadaso.

Qhita. Hor he swmaride tera bueno in mejor the of mio.

Maria. Si señaras el nuebo alguacil, es mu than lamas, porthe derde are mu mes the not kasemas, el ba á la komprar me llebar el choeslate ai la kama; el se plandha uska. misas, it ti io kiero, me jlancha las enaquas, axi es he mustro matrimanio uslichero, por the tenemas 30.000 rades puestos a ródtes. La abitasción mestra, ostai biew anureblexara; ae la entro i sol. go rolar hair à danoe kiero i noaic men Dire nada, is abra, knu do ke ganamid han el expecos.

han et amplea, posemas parar niva bida Duster. The dichora es Of nientras he is bilo ha mo nua esklaba, sin poder salir sala os trana, sufriendo has impertinenrias de las as emand, the 97 abros las kanane de list tas, pers ns hanyerevoe sus intenziand i mañas. Por lo cemais, bibo desacagadar, poo que troutands kaw rehured is despies tenaremos mas intereses par be an eredrado los das emand par partes iquales doce mil auros, de un tio tranal he are patso murio en la Oméritia.

Maria. Diga \%P. señara, si su huñaroo nuvere, senia para OPal toda la ereuxia.

Thesta. Si señora, mi manido is is eredames los

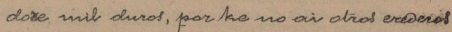
ferzatos.

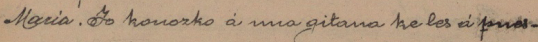
to loktos kampletamente à barios ambies, i si GP. Aivere, La llamaré para ke tomalich

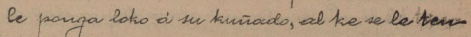

- dria ke llebar di un manikemio, i enton. xes teria para ofol toda la eremsia. . 


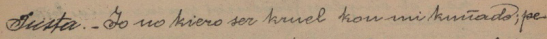

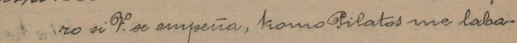
ro las manos.

Hacía. Orodar nada, hav Plamarí di la gitoma para

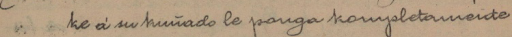
loko.

- Pasians. De buerra me è eskapaso, en la karral me keriom peogar bergaras

Stesta. Pero ambre, Ramán ikama te as eska. .... pads?

Pomán. Co te kantarié hrego a valas i despario. Tretar. Grimero ma tienes he derivo kamo as ro. to la botella en el krapé, par ke se he tiene. ke pagar es perjuiris al kafetero, st alkamarero.

(Debe estarf end rinchás all curenaxis un

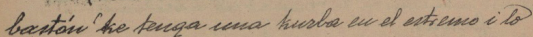
rage Penaini).

Porivan. Bes este bastón. purs atro ignal temia 2. is knando no an echads press en el hafé. Lo tenha atrabesado por dectrás kan las dos brazos, $i$ al doar media buelta para mirar à atro laso, la curba del bastón
$-25$.

a' engarhaso à la botella llena de aquar i ras, a taids la botella of suclo, kamo es to ara, is se a edio añikos, is a an moja. do mos parrahianos, haw los the e' tenlabo kre andar a sopapas.

Trecta. Bribon, Ponnón, nira kamo nos as mo jado a la veñora i i uni las faldas i las enaquas (se har lebautar).

Penciun. Espesar mu proko, he sakaré la mahima instantonea para retratarlas.

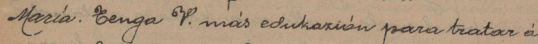
las suraras. (be da un rapoppo en be er padalajel fotógrapto.)

Arista. Aarar tro nolnbiera odurredo esto, is debia de aber ponesto en el olizio, el he ns le sa. karian a Ravian tow pronto de la hasnel.

Otesta, Bamos à ber knenter abra hamo te as eshapado de la karral.

Pemian. Berás lo he i pasads. Ol ke me haw duria ev el kamino, he é robado un afizio tre thebaba en eh bolsillo de lar a. merihanar, el kre lo é presentadols al

\section{$-26$.}

Olkaise, hien lo à leialo i à prequntacio trieur era el konousiao, à lo he è hantesta do ke el otro i ke is era el hanourator, $i$. at ke me à akompanaho, erev mu lako? barrachs, is presdido. Otros dot kan bergas, atremprañabion al alkaide, tien à dads orden para twe le hastigarian al atro, how nigon, freste i a la kabera, astar ha houfie-

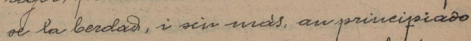
á pegarle berganes; à rodabo su histeras por el sucls, is alli se à healaso quitands? he is no estoi lako, the io no estor barra. cho, io me eskapado, iv el otro alliv 1 is. hedorso gritando; ese, exc de la hapa es el ken está hobo $i$ berracho.

Aaria. Or, ain ha me desmais; uni buen marido resiew harado is sin kulpa estio reni biends bargarel; ai, ai, he sin remedio is de sentimiento me mueso. ( Fe deja haer ean d sudo traen forehavisioner.

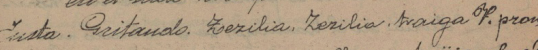
to a navi binarge is llame tioñis Médiko he bibe en el piso princisal de es.
$-23-$

tea hara

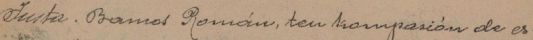

sti ta pobre nujer, sugetala las manos. is la 5. Anclariné las paies.

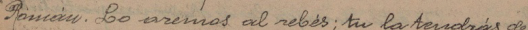
less mandis is de los prees para he wo beya a llomar a las gitomarpara ke me ponga hompletamente lako, hans lo a dicho huando is entraba por exa puen. tha.

Eevilia. Soñona, a hi esta el aqua rebudla kon binagre isia biene el Médiko; en ha eshalcra lo é enhoutrado.

Fusta. Foerilia, troxiga M. un kawdere tian bela enrenaida para kuando benga de Héchino.

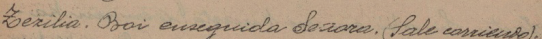
tista. Bamos Poncinv, noja las monas i la kavia de esta breula mujer tran el agua i binagre he estion solare exen me. tre.

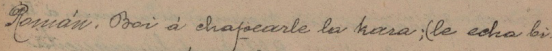
nazer trow lot dectas o' haw nua es 
$-28$.

parja.

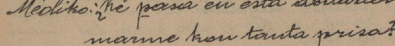

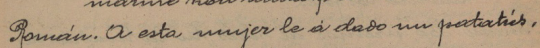
ino rabemos hurasla.

Héditu. Ober si e la puese poner sentada. Ppmíur. Li reños, se larrentard annque ella no hiera i no pueva. (Lbarriecta).

Níaliho. Oberesito ma har.

Verilia. Otri está i alumbrare' (axib ere.)

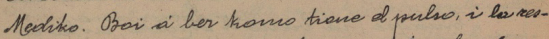
pirasión (pares el ocido wi el precho derpuein de toverel el prubles).

Néditho. Esta enferna estai nuy grabe is se le tiene the akastar ensequidea.

oresta. Si, aki en mi tmeasto is en mi ka. ma he está merka; baner of Nédike, 9. sactenga la trabera; terilia le hogerá del medic iv de las pies. Ponán ahmibrana haw el kanoelero; arenal ba frevra i untiempo; à la mua, $a$ las dos i vi las tres. (Blebacu os la myenesta.

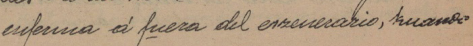

29

dire

Sécilisa. Estar munger está muerta; ticue los ojol bueltos i era mejor llanar a' las trueas para ke le kuntew el gari gari. i los nes pones.

Nédiho. Eodabia la enferma respira; band a la kama haw ella: (Le La bebaw iPenow exlumbera par delante). Olas prohot sequnder, entra quitercudo el

Dthalde. Esfrosa, Eterilia, bloquarila, the si trieres, las tres se an domido, ci pesal de ke mi esposa à tamado kafé, ke es un remedis efikare houtra el sueno.

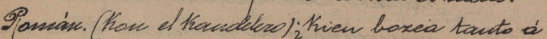
esta ora tan intaypestiba en hasa old alkalde?

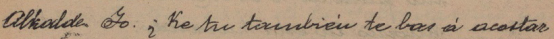
tran esa uro, huoundo debias estar enta haved.

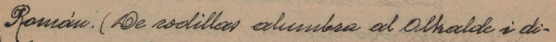
se (Perdoname emano, it the dire lo the à pasabo.

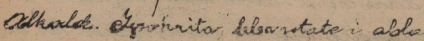

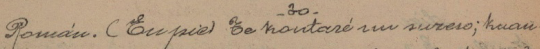
do el aloquaxil me llebaba a la tierrad, del bolvillo de ha amorithana, le é hi:tado un ofizio; se lo e'dado al olkaib,

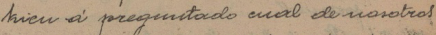
era el kondunido, a lo the è kautastodo ke el atro it he so era el havourtor por aber prescutrado el ofiris, i ensequida, al atro, amprinnipias do a pegarle ber. garos; à rodavo su chistera parel sue lo, knowoo pitaba, io no estai liho, ni bonadhs, ivo entannes me es es. trapado. Esta numa relarián la é manfertado en preservicia de sei rima jor ke aki estaba, wi la ke le al doco mu pataties, i el Nédico en ese otio kivarto la está kurando.

calkadal. Exer umirfane, un tirinuinal, boi à dar pasto al Ministro de la Oobemanión, pera he par nui cho tiempo te se licbe a la havad; i despries bendra mi Olouaril á sahnairte buenos bastovard. De on estrapas (fale intrainodeade).

Pornain. Ploriaro San Poque, ar nu milagro para he no me leben d la karrel ni

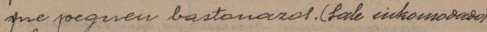
(Eutra dista)

Ducitos. Honcin, ia está la enforma frera de peRigro, i pranto bendri kaw el Nédiks abl brano.

Raman. (Eufadard) a la enforna, al Médiko i ati, ia es pueaer ledas el Diablo.

Insta. Pero huñado, the te parsa paravestors enfavaso.

Pasnin. (Are he llaca) Mi emeno akaba de salis nui inkomodado parkels édi cho todo lo ke aki i ew la karnel a parsaso. A ids à dar praste al Mobenuasar in al Ministerio de la Mobernaxión, para ke prara uncho tiempo nue lebewá la trarsel i despnés a' didio he beredrá el alquasil à progame bastonarer (hique Ulavoualo).

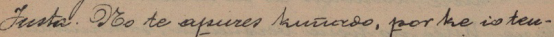
an-indlonemria ervlos Atiniaterios is al 
$-32$.

Irexidente par el telefors que tenencal en hersa, dizé he la roticra de las batelias: sids hasud, is me parere ke se to perdonara'; sin embargoisu embargo the pueves arerle saber, he no eres treefperble, al Oobernadar6, parra the no le agan trans. a' the emano.

Ponán. Enmona Tustai, tw exes brena, por

he por mi te interesas dednoame áwmo, $i$ artes de he nas reparemor pasa prantihers diligensian, perniteme kete de un abrara.

Tuste. Obranonne it tew áminuo (se abreran huando of Neicho i Haría eutraw all braro.

- Hacía Anvar anda, las dos tuñados se alma naw i si lo llega ai raber el Oekadeles, se ba amacr ma dacurar i pegará bastanarad.

Gendiu. Leñara, no tanga \%. envidia por ke si hiere, tambieiv la abranaré.

Maria. Hstes no tiene beroinewra, so hounalla, (le prepa otra bes che las expoddar) atren-
$-33-$

bense à fudir un abrare, à mua hara. Dar les ke ai de predir bengaurar auto lof Eribunales, par los bergaxs he injusta. mente i nesibids mi brew maride.

Pasnam. Seinona, porsone of: estai in otras ligerenas, he boi a prosatitian diligensiod. (fate)

Cresta. Os. Nédiko; ia the la enferma está frera. de hiviobo, hamberará el he beba para kambalenenxia of buen bive de of alse perias he tengo en trasa.

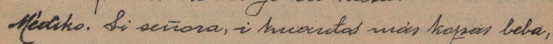
mais ráfsida rerá la kambalenenria.

Lusta. Tr la hriada no si hiew la dinpieste; bai a trace el haro i la batella (Sale. ha batella debe de nes de nqua hav kolor de bino pare he revinta tha exfernal

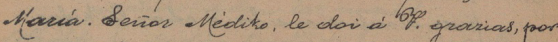
aber buraso mi eifermedas en padio hienypo.

Midiko. Prede op. dar quarias à la rienria mielica, the en Éspaña, como ruto. das prartes está adalantanes fopra toDar blare de entermedocies.

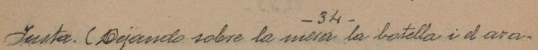

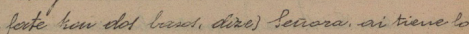

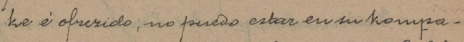
nia por the tringo the ablar pon ch Belefono ke teneruas en esta in kasa. (fale) . 5

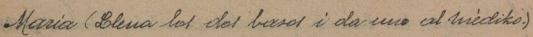
Maria. Dramas of Midithe, uga el fabor de bes? ber cute baso del buew hino dhe Tolde:pounas.

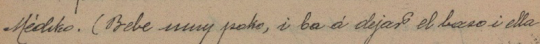
lo kage.

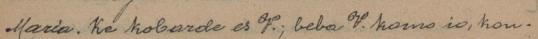

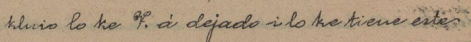
otro bass (ari lo are i enrequida sepere.

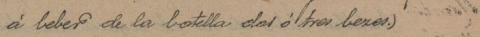

- Hédiko. Señarar the of. se ba di embriagas i despries a' mi re me ha à enhas la kulpas; baiei ohultar la botella en la editarión

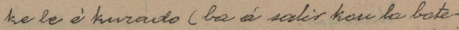

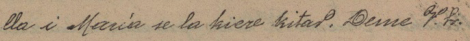

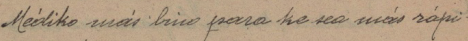
da la kavbalerenxia.)

Míaike. T6o puede ser, por he mo seiane la bote.
Slav

$-35$

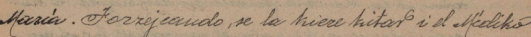

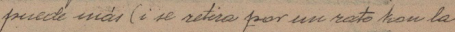
botellas.

Eutrow of Altade iel Olguaril haw un brars holgado de une paciundo del truello.

Alguarif. Eodabia bibe mi mujor, ha he me on didno ho estaba muerta, bew exposa he te dev un deraro.

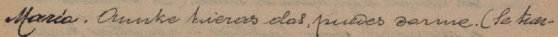
re homo borradha).

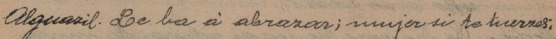
the as bebido algu.

- Lavia. Pevá la debilidas ha tengo. (Gutra-d

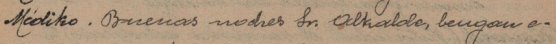
sos sicho; se dam las monas.

Odkadde. He. Médicho, tanto de bueno par esta un kasa.

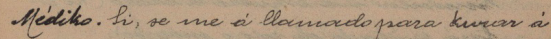
exta Peñona, he ai extá fruzar de huida. do, i sique hien eur la kanbalorensia.

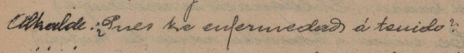



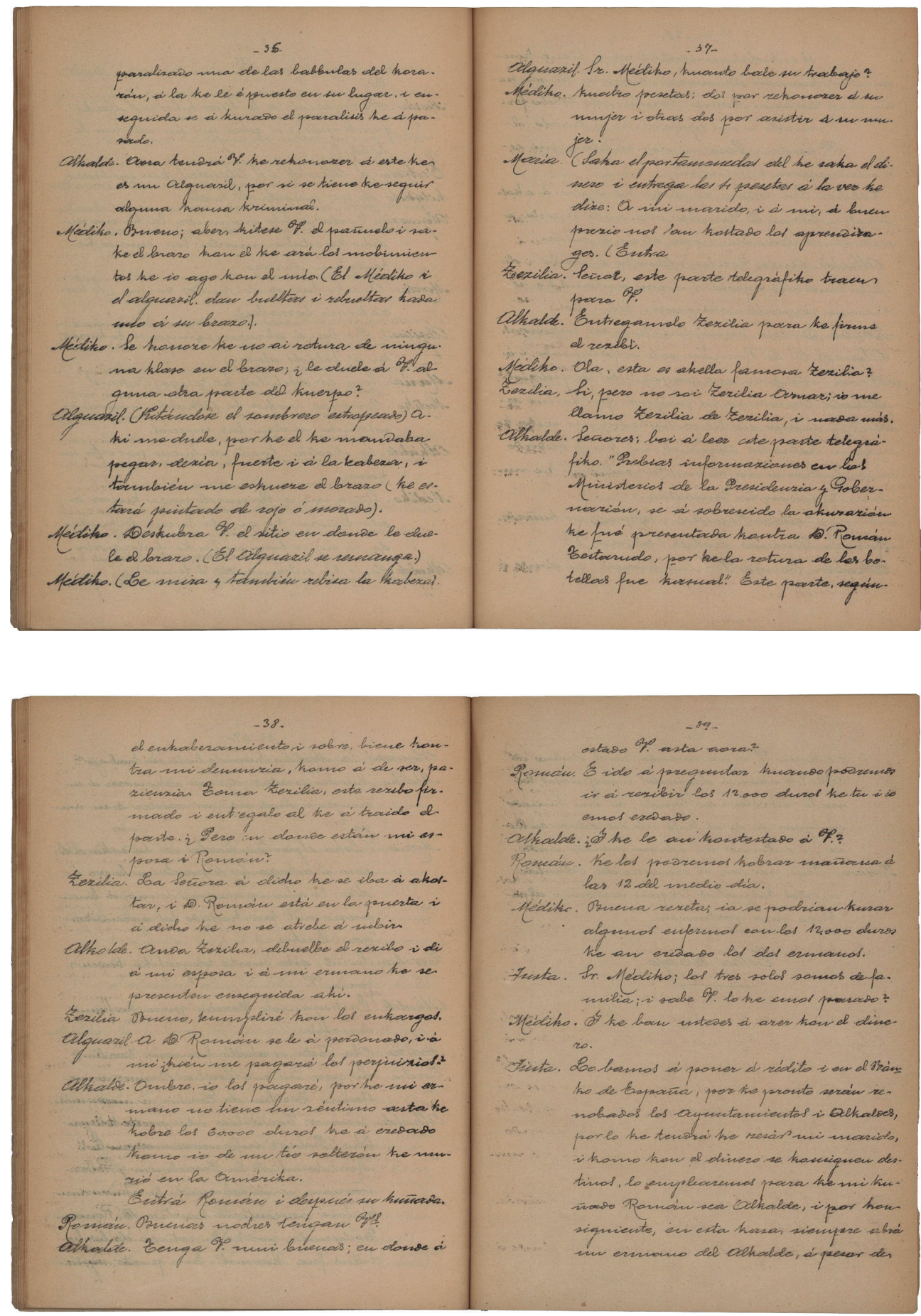


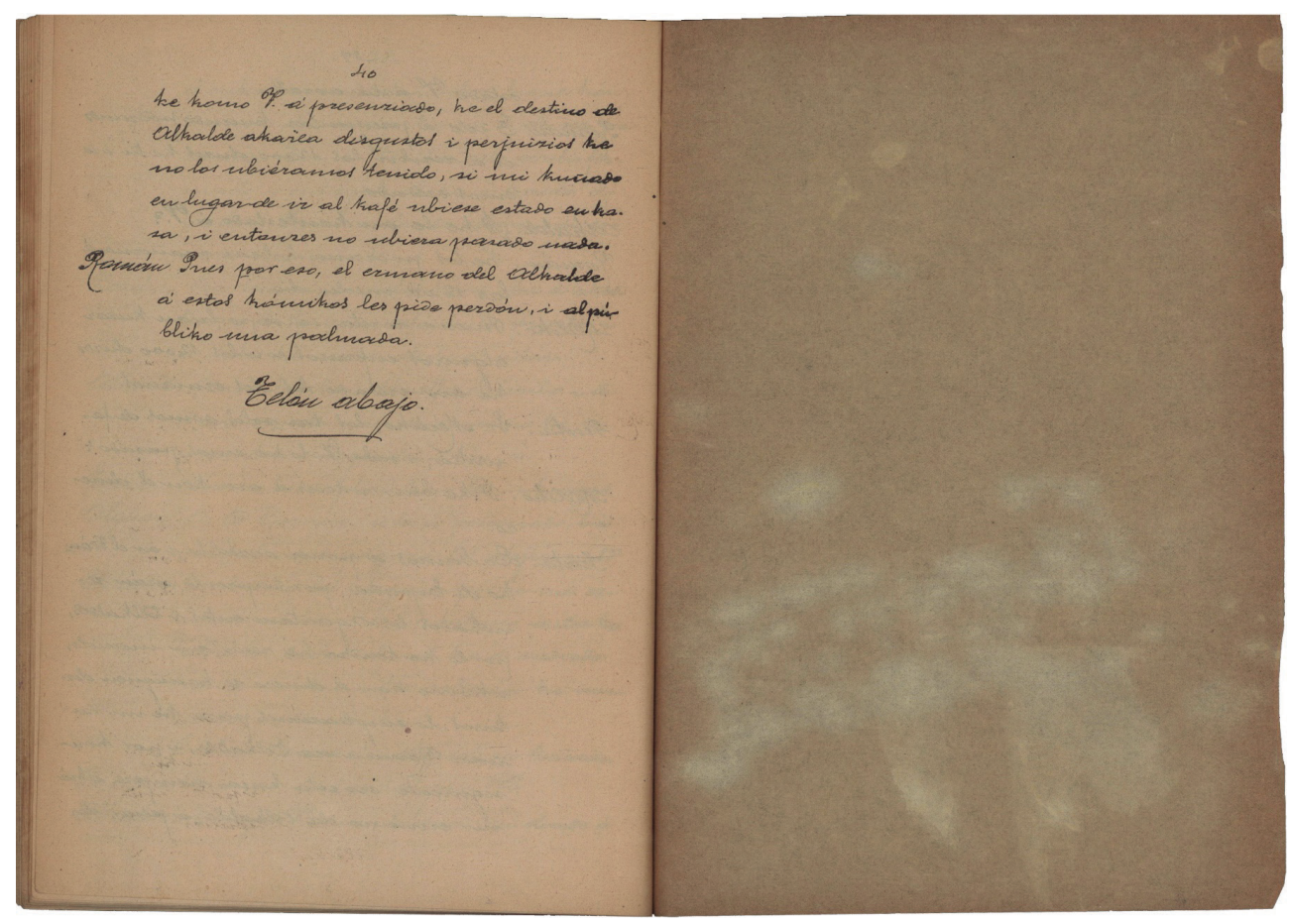

